\title{
The Angular Broadening of the Galactic Center Pulsar SGR J1745-29: A New Constraint on the Scattering Medium
}

\author{
Geoffrey C. Bower ${ }^{1}$, Adam Deller ${ }^{2}$, Paul Demorest ${ }^{3}$, Andreas Brunthaler ${ }^{4}$, Ralph Eatough ${ }^{4}$, \\ Heino Falcke ${ }^{5,2,4}$, Michael Kramer ${ }^{4}$ K.J. Lee ${ }^{4}$, Laura Spitler ${ }^{4}$
}

\begin{abstract}
The pulsed radio emission from the Galactic Center (GC) magnetar SGR J1745-29 probes the turbulent, magnetized plasma of the GC hyperstrong scattering screen through both angular and temporal broadening. We present the first measurements of the angular size of SGR J1745-29, obtained with the Very Long Baseline Array and the phased Very Large Array at 8.7 and $15.4 \mathrm{GHz}$. The source sizes are consistent with the scatter-broadened size of Sagittarius A* at each frequency, demonstrating that SGR J1745-29 is also located behind the same hyperstrong scattering medium. Combining the angular broadening with temporal scattering obtained from pulsar observations provides a complete picture of the scattering properties. If the scattering occurs in a thin screen, then it must be at a distance $\Delta \gtrsim 5 \mathrm{kpc}$. A best-fit solution for the distance of a thin screen is $\Delta=5.9 \pm 0.3 \mathrm{kpc}$, consistent with being located in the Scutum spiral arm. This is a substantial revision of the previously held model in which the scattering screen is located very close to the GC. As also discussed in Spitler et al. (2013), these results suggest that GC searches can detect millisecond pulsars gravitationally bound to Sgr A* with observations at $\gtrsim 10 \mathrm{GHz}$ and ordinary pulsars at even lower frequencies.
\end{abstract}

Subject headings: pulsars: general, pulsars: individual(SGR J1745-29), black hole physics, scattering

\footnotetext{
${ }^{1}$ Astronomy Department and Radio Astronomy Laboratory, University of California, Berkeley, 601 Campbell Hall \#3411, Berkeley, CA 94720, USA; gbower@astro.berkeley.edu

${ }^{2}$ ASTRON, P.O. Box 2, 7990 AA Dwingeloo, The Netherlands

${ }^{3}$ NRAO,520 Edgemont Road, Charlottesville, VA 22903-2475

${ }^{4}$ Max-Planck-Institut für Radioastronomie, Auf dem Hügel 69, D-53121 Bonn, Germany

${ }^{5}$ Department of Astrophysics, Institute for Mathematics, Astrophysics and Particle Physics (IMAPP), Radboud University, PO Box 9010, 6500 GL Nijmegen, The Netherlands
} 


\section{Introduction}

A pulsar in orbit around Sagittarius A*, the black hole at the center of the Galaxy, has the potential to provide a powerful probe of general relativity and the structure of space-time in the strong field limit (e.g., Backer \& Hellings 1986; Wex \& Kopeikin 1999; Pfahl \& Loeb 2004; Cordes et al. 2004; Liu et al. 2012). There have been numerous searches for such a pulsar without success (e.g., Kramer et al. 2000; Johnston et al. 2006; Deneva et al. 2009; Macquart et al. 2010; Siemion et al. 2013; Eatough et al. 2013b). Searches of this kind have been assumed to be challenging due to the presence of very large temporal broadening of radio pulses due to strong interstellar scattering (Cordes \& Lazio 1997) that is inferred from the very large angular broadening seen for Sgr A* and other GC sources, with sizes of $\sim 0.5$ arcsec at $1.4 \mathrm{GHz}$ (van Langevelde et al. 1992; Bower et al. 2006). The GC hyperstrong scattering screen has been modeled with a turbulent, ionized thin screen at a distance from the GC of $\Delta=133_{-80}^{+200}$ pc (Lazio \& Cordes 1998). The large angular broadening and the proximity of the screen to the GC implies a temporal broadening of pulsed emission of $\gtrsim 10$ seconds at $1.4 \mathrm{GHz}$, eliminating any opportunity to detect pulsars at low frequencies. Pulsar searching at higher frequencies, however, is less sensitive due to the typical steep pulsar spectrum. Detection of a pulsar in the GC provides us with the opportunity to simultaneously probe the angular and temporal properties of the scattering medium.

The pulsar SGR J1745-29 was discovered serendipitously through its X-ray emission by the Swift telescope (Kennea et al. 2013). Subsequent NuSTAR observations detected periodic flux variations with $P=3.76 \mathrm{~s}$ and a hydrogen absorption column characteristic of a GC location (Mori et al. 2013). Chandra observations demonstrated that the source was offset from Sgr A* by approximately 3 arcseconds, or a projected separation of $0.1 \mathrm{pc}$ (Rea et al. 2013). The measured period derivative implies a magnetic field $\sim 10^{14} \mathrm{G}$ and a characteristic age of $9 \mathrm{kyr}$. The spin down power is inadequate to account for the X-ray flux, identifying SGR J1745-29 as a magnetar (Thompson \& Duncan 1995).

Radio pulsations were detected from SGR J1745-29 at frequencies ranging from 1.4 to $20 \mathrm{GHz}$ (Eatough et al. 2013a; Shannon \& Johnston 2013; Spitler et al. 2013). The radio observations confirm the X-ray pulse period and period derivative. They also determine the dispersion measure $\mathrm{DM}=1778 \pm 3 \mathrm{pc} \mathrm{cm}^{-3}$ and the rotation measure $R M=-66960 \pm$ $50 \mathrm{rad} \mathrm{m}^{-2}$. These two measures of the line of sight plasma properties indicate that SGR J1745-29 is very likely to be physically close to Sgr A* and shares many of the same line of sight characteristics; the DM is consistent with predictions of the galactic electron density model (Cordes \& Lazio 2002).

We present here the first very long baseline interferometric (VLBI) observations of SGR J1745-29 obtained with the Very Long Baseline Array (VLBA) and the phased array out- 
put of the Karl G. Jansky Very Large Array (VLA). These observations provide a direct measurement of the angular size of SGR J1745-29. Coupled with temporal broadening from pulsar observations (Spitler et al. 2013), we are able to place a new set of constraints on the geometric model for hyperstrong scattering screen. We present our observations and data analysis in $\S 2$ and discuss results in $\S 3$,

\section{Observations and Data Reduction}

SGR J1745-29 has been observed 4 times using the VLBA + phased VLA as part of a campaign to measure the proper motion of SGR J1745-29 (project codes BB336, BB337; Table 1). Each observation was 6 hours in duration and used Sgr $A^{*}$ as a delay and phase calibrator. In the first observation, the VLA was in the D configuration and the synthesized beam was large enough to encompass both SGR J1745-29 and Sgr A* simultaneously. For this observation only, an external phase reference calibrator (J1752-3001) was observed for 1 minute every 5 minutes. For all other observations, the VLA synthesized beam was too small to include both Sgr A* and SGR J1745-29 simultaneously, and so we performed a nodding cycle of total duration 2.5 minutes, with 36 seconds spent on Sgr A* and 96 seconds on SGR J1745-29.

Due to scatter-broadening, Sgr A* appears as a Gassian with major axis size $1.32 \lambda^{2}$ mas at a wavelength $\lambda \mathrm{cm}$ (Bower et al. 2006). Nearby background sources are also scatterbroadened, making it impossible to calibrate the longest VLBA baselines. We were unable to calibrate the Saint Croix, Mauna Kea and Hancock stations; only short timeranges of useful data were obtained for Brewster, North Liberty and Owens Valley. The first observation which included an external calibrator provided better results for the more distant stations than the subsequent observations. Difficulties with array phasing resulted in the loss of 2

Table 1. Observations of SGR J1745-29

\begin{tabular}{cccc}
\hline \hline $\begin{array}{c}\text { Epoch } \\
(\mathrm{MJD})\end{array}$ & VLA configuration & $\begin{array}{c}\text { phased VLA resolution } \\
\left({ }^{\prime \prime}\right)\end{array}$ & $\begin{array}{c}\text { Observing frequency } \\
(\mathrm{GHz})\end{array}$ \\
\hline 56422 & $\mathrm{D}$ & 8.5 & $8.540-8.796$ \\
56444 & $\mathrm{C}$ & 2.6 & $8.540-8.796$ \\
56473 & $\mathrm{C}$ & 1.5 & $15.240-15.496$ \\
56486 & $\mathrm{C}$ & 2.6 & $8.540-8.796$ \\
\hline
\end{tabular}


hours of data from the second epoch, reducing sensitivity and uv coverage.

Before VLBI correlation proceeded, the phased VLA data were analysed to provide contemporaneous timing information to enable pulsar gating (e.g. Deller et al. 2007). For VLBI observations, the phased VLA records baseband voltage in VDIF format (Whitney et al. 2009), which we then processed using DiFX correlator tools (Deller et al. 2011) and the DSPSR software (van Straten \& Bailes 2011). Then, for each observation of SGR 1745-29, a 256-channel coherently dedispersing filterbank was applied to split the full band into 1$\mathrm{MHz}$ wide channels, and remove interstellar dispersion at the known DM of the pulsar. This was followed by full-Stokes detection and averaging into 4096-bin single pulse profiles ( $\sim 1 \mathrm{~ms}$ time resolution). By measuring the amplitude of the VLA $10 \mathrm{~Hz}$ switched noise calibration signal as a function of frequency and polarization, we were able to correct for differential gain and subtract the switched noise signal from the filterbank data. The calibrated, $10-\mathrm{Hz}-$ removed pulse profiles were averaged into 5-minute integrations for MJD 56422 and 90-second integrations for following epochs, from which we generated pulse times of arrival (TOAs) in four 64-MHz subbands using the PSRCHIVE software (Hotan et al. 2004; van Straten et al. 2012). The pulse reference phase, average pulse period and DM for each observing session were measured using the TEMPO software 1 . This information was then used to determine an on-pulse gate for VLBI correlation.

The average pulse profile (Figure1) determined from our VLA data consists of two broad features with an overall width $\sim 150 \mathrm{~ms}$, and a low-level trailing component extending another $\sim 100$ ms. However, individual pulses vary dramatically in shape, and exhibit structure on ms timescales. The width of these subpulses can be used to constrain the temporal scatter broadening affecting the pulsar. To quantify this, we reprocessed our MJD 56486 data into 32768-bin single pulse profiles (0.1 ms time resolution). We computed the average autocorrelation function (ACF) of all the high-resolution pulses using an on-pulse window of 0.4-0.6 turns pulse phase. To remove potential bias due to any non-pulsar noise processes we subtracted the average ACF measured in two adjacent off-pulse windows of identical length (pulse phase $0.2-0.4$ and $0.6-0.8$ ). The ACF shows a sharp, fully resolved component with a $1 / e$ width of $1.9 \mathrm{~ms}$. Temporal scatter-broadening in excess of this value would push power to larger lags, so we interpret this as a conservative upper limit on the scatter-broadening timescale at $8.7 \mathrm{GHz}$. This upper limit is consistent with a measured timescale at $8.36 \mathrm{GHz}$ of $0.3 \pm 0.4 \mathrm{~ms}$ (Spitler et al. 2013).

The VLBA and phased VLA data were correlated using the DiFX correlator (Deller et al. 2011). Three correlator passes were employed:

\footnotetext{
${ }^{1}$ http://tempo.sourceforge.net
} 
1. a gated pass using the ephemeris information derived from the VLA and the position of SGR J1745-29 for all scans on SGR J1745-29 or Sgr A* (the "gated" pass);

2. an ungated pass using the position of SGR J1745-29 for all scans on SGR J1745-29 or Sgr A* (the "ungated" pass); and

3. an ungated pass using the position of Sgr A* for all scans on SGR J1745-29 or Sgr A* (the "calibrator" pass).

A simple pulsar gate of width approximately $150 \mathrm{~ms}$ was used, boosting the $\mathrm{S} / \mathrm{N}$ by a factor of $\sqrt{3.76 / 0.15} \sim 5$ on SGR J1745-29 in the gated pass.

Data reduction used the AIPS software package (Greisen 2003) with the ParselTongue interface (Kettenis et al. 2006). Visibility amplitudes were corrected for the logged system temperatures (and the nominal correction for the VLA, since no logged information was available). Delay calibration was performed using the ungated dataset with J1752-3001 in the first epoch and Sgr A* in subsequent epochs. Phase and amplitude self calibration with a timescale of 20 s was performed using $\mathrm{Sgr} \mathrm{A}^{*}$. The solutions and visibilities were flagged to remove outliers and $\operatorname{Sgr} \mathrm{A}^{*}$ was imaged using natural weighting with visibility weights raised to the power 0.5 to reduce the dominance of the VLA baselines. The calibration and flags were then applied to the gated and ungated correlator pass data.

Imaging at the position of SGR J1745-29 requires an accurate subtraction of Sgr A*, which is only several hundred synthesized beamwidths away and around 5000 times brighter than SGR J1745-29 in the ungated image. This is particularly problematic in the second and subsequent epochs, because Sgr A* moves through the sidelobe pattern of the phased VLA during observations of SGR J1745-29, suffering large gain variations. We remove Sgr A* by visibility peeling. Phase and amplitude self-calibration solutions were determined for the VLA using the ungated pass visibilities and the Sgr A* model derived from the calibrator pass. This calibration was applied to the gated pass dataset and the model of Sgr A* from the clean image was subtracted; then, the calibration was reverted using the AIPS task CLINV. In this way, optimal signal-to-noise was obtained for both the peeling solutions and the final gated image.

For the 3rd epoch at $15 \mathrm{GHz}, \mathrm{Sgr} \mathrm{A}^{*}$ is further into the sidelobes of the phased VLA, making the effective VLA gain in the direction of Sgr A* during the scans on SGR J1745-29 even lower and more rapidly variable. Unsurprisingly, this epoch suffered from much worse dynamic range issues resulting from the imperfect subtraction of Sgr A*.

After imaging with IMAGR using the same weighting scheme the sources were fitted using the AIPS task JMFIT. The resulting deconvolved, scatter-broadened sizes for SGR 
J1745-29 and Sgr A* are listed in Table 2. Images of SGR J1745-29 at 8.7 and $15.4 \mathrm{GHz}$ are shown in Figure 2, The $15.4 \mathrm{GHz}$ image clearly shows the residual effects of imperfect calibration and subtraction of Sgr $\mathrm{A}^{*}$, and highlight that the fitted values at this frequency should be interpreted with caution.

\section{Discussion and Conclusions}

The measured size for Sgr A* is consistent with past measurements of the $\lambda^{2}$ scattering law. Bower et al. (2004) obtained a size of $17.6 \pm 0.3 \times 8.5_{-1.9}^{+1.3}$ mas in position angle $82 \pm 4$ degrees at a frequency of $8.4 \mathrm{GHz}$. Scaling by the $\lambda^{2}$ law to $8.7 \mathrm{GHz}$, the observation frequency of these new measurements, we find a size $16.4 \pm 0.3 \times 7.9_{-1.8}^{+1.2}$ mas, which is in agreement with the measured sizes for Sgr A* in Table 2. SGR J1745-29 has an angular size that is statistically indistinguishable from that of $\mathrm{Sgr} \mathrm{A} \mathrm{A}^{*}$ at $8.7 \mathrm{GHz}$. The small difference in the $15.4 \mathrm{GHz}$ fits is almost certainly due to residual calibration/subtraction errors. The similarity between the angular broadening seen for SGR J1745-29 and Sgr A* confirms that SGR J1745-29 must be close to the GC and share a similar line of sight scattering medium. In particular, it rules out the possibility that SGR J1745-29 resides in front of the hyperstrong GC scattering screen, which would otherwise be a possible explanation for the low temporal broadening discussed below.

The temporal scattering, $\tau$, is related to the angular broadening due to the longer path length required for scattered photons to reach the observer. The time delay is proportional to the path integral of the solid angle and, therefore, has a different weighting with distance than the angular broadening angle. For the same angular broadening, the temporal broadening is much larger if the scattering occurs close to the source. The angular and temporal scattering scales for a thin scattering screen are related by the following equation:

$$
\tau=6.3 \mathrm{~s} \times\left(\frac{D}{8.5 \mathrm{kpc}}\right)\left(\frac{\theta_{1}}{1.3 \operatorname{arcsec}}\right)^{2}\left(\frac{D}{\Delta}-1\right) \nu^{-4},
$$

where $D=8.3 \pm 0.3 \mathrm{kpc}$ (Genzel et al. 2010) is the distance to the GC, $\Delta$ is the distance from the GC to the pulsar, and $\nu$ is the observing frequency in GHz (Cordes \& Lazio 1997). $\theta_{1}$ is the observed angular size extrapolated to a frequency of $1.0 \mathrm{GHz}$ using a scaling of $\nu^{-2}$. This equation assumes a symmetric scattering medium, which is accurate to a factor of 2 in this case. The effects of anisotropy on scattering tend to be small (Chandran \& Backer 2002). We use the geometric mean of the major and minor scattering axes at $8.7 \mathrm{GHz}$ for our calculations, $\theta=12.5 \pm 1.2$ mas, implying $\theta_{1}=945 \pm 91$ mas.

Lazio \& Cordes (1998) determined $\Delta=0.133_{-0.08}^{+0.2} \mathrm{kpc}$, implying that $\tau$ ranges from 18 to $120 \mathrm{~s}$ at $1.4 \mathrm{GHz}$ for the $1 \sigma$ range of $\Delta$. The maximal broadening occurs for the screen 
closest to Sgr $A^{*}$. Thus, this estimate for $\Delta$ is clearly inconsistent with the detection of pulsed emission at frequencies below a few $\mathrm{GHz}$ in a pulsar with even a period as large as $P=3.7 \mathrm{~s}$.

We combine our angular broadening measurement with temporal broadening measurements from Spitler et al. (2013) and our $8.7 \mathrm{GHz}$ VLA data to constrain $\Delta$ (Figure 3). Solutions are permitted for values of $\Delta$ where $\tau$ falls below the measured pulse width. We consider only measurements at frequencies $<10 \mathrm{GHz}$; above this frequency, the scattering timescale becomes much smaller than the intrinsic pulse widths. A thin screen solution is consistent for all frequencies with $\Delta \gtrsim 5 \mathrm{kpc}$. A best-fit solution using a scattering time scale at $1 \mathrm{GHz}$ of $1.3 \pm 0.2 \mathrm{~s}$ (Spitler et al. 2013) for a thin screen scattering distance is $\Delta=5.9 \pm 0.3 \mathrm{kpc}$.

This estimate for $\Delta$ is significantly larger than the upper bound on $\Delta$ set by the $\mathrm{OH} / \mathrm{IR}$ star distribution and the number counts of extragalactic background sources Lazio \& Cordes (1998). Recent results provide a counter to each of these arguments. First, while a subset of the $\mathrm{OH} / \mathrm{IR}$ stars within the central 0.5 degree do show very strong scattering, scattering angles that are smaller by only a factor of two are seen as far away as $6 \operatorname{deg}$ from Sgr A*. The discovery of patchiness in the scattering medium (Roy 2013) suggests that the line of sight towards Sgr A* may not be as unique as previously stated and that the assumption of a single uniform screen for Sgr $\mathrm{A}^{*}$ and the $\mathrm{OH} / \mathrm{IR}$ stars is not sufficient. Second, new extragalactic background source counts indicate that deficit in source counts previously found may not be as significant as previously estimated. Lazio \& Cordes (1998) reported a significant deficit in 1.3 and $1.7 \mathrm{GHz}$ surveys of the GC, which was accounted for by very strong scattering that leads to angular broadening of $\gtrsim 100^{\prime \prime}$ for extragalactic sources. However, Roy (2013) recently used 154 and $255 \mathrm{MHz}$ extragalactic source counts to show that only a small fraction of sources are missing due to scattering. Additionally, other scattering sources that are either very close to or more distant from the GC could affect extragalactic background sources without influencing Sgr A* or SGR J1745-29.

Additionally, the detection of Sgr A* at $330 \mathrm{MHz}$ demonstrates that the free-free optical depth towards Sgr A* is $\gtrsim 1$ (Nord et al. 2004). As van Langevelde et al. (1992) demonstrate, a low optical depth requires that the scattering screen be located at a distance $\Delta>0.1 D \sim$ $0.8 \mathrm{kpc}$, consistent with our much larger distance.

Since the hyperstrong scattering medium is not local to the GC, it must be located in one or more scattering screens along the line of sight to the GC. A few other lines of sight, such as towards NGC 6334B and the Cygnus region, have comparable or larger scattering measures, which are argued to be due to random superposition of very turbulent clouds (Wilkinson et al. 1994; Trotter et al. 1998; Desai \& Fey 2001). The characteristic turbulent 
clump size in the interstellar medium of 50 pc (Fey et al. 1991) has a sufficiently large angular size at $5.9 \mathrm{kpc}$ to be responsible for Sgr A*, SGR J1745-29, and many of the OH/IR masers. A distance of $5.9 \mathrm{kpc}$ from the GC falls within or close to the Scutum arm, which hosts giant molecular clouds and HII regions that could be responsible for the scattering (Reid et al. 2009).

Radio continuum measurements towards the GC constrain the physical conditions of the scattering screen with a relationship between $\Delta, l_{0}$, the outer scale of the turbulent electron density (in pc), and $T_{e}$, the electron temperature (in K; Lazio \& Cordes 1998):

$$
\log l_{0}^{2 / 3} T_{e}^{-1 / 2} \approx-7.0+2 \log \frac{\Delta}{150 \mathrm{pc}}
$$

For $\Delta=5.9 \mathrm{kpc}$, we find solutions at typical warm and hot ionized medium temperatures $\left(T_{e}=10^{4}\right.$ and $\left.10^{6} \mathrm{~K}\right)$ of $l_{0}=10^{-3} \mathrm{pc}$ and $l_{0}=3 \times 10^{-1} \mathrm{pc}$. These estimates for the outer scale are consistent with the lower limit of $\sim 3 \times 10^{-2}$ pc found from other galactic measurements (Armstrong et al. 1995). Thus, the scattering screen appears to have properties that are consistent with other turbulent regions in the interstellar medium.

The scatter broadening may occur in a thick screen or other unconventional geometric model in which Equation 11 does not hold. Spitler et al. (2013) discuss thick screen models and the evidence that may support that interpretation. Our results demonstrate, however, that the angular broadening of SGR J1745-29 resembles that of Sgr A* and we have very strong constraints on that image: the angular size scales as $\lambda^{2}$ and the angular size at a given wavelength is very well parametrized as a Gaussian (Bower et al. 2004). These facts point to a conventional thin screen model for the pulsar scattering.

The absence of strong temporal broadening for the SGR J1745-29, coupled with clear evidence that SGR J1745-29 sees the same scattering medium as Sgr A* via the angular broadening, makes clear that millisecond pulsar companions to Sgr $\mathrm{A}^{*}$ can be detected at frequencies $\gtrsim 10 \mathrm{GHz}$, while ordinary pulsars can be detected at frequencies of a few $\mathrm{GHz}$. Searches at $10 \mathrm{GHz}$ will face temporal broadening of less than a millisecond, making observations sensitive to even the fastest spinning pulsars orbiting the black hole. It is surprising that past attempts to search for GC pulsars have not been successful given the expected large number of compact objects near Sgr $\mathrm{A}^{*}$ and the broad frequency range of those surveys (Macquart et al. 2010, and references therein). The detection of a pulsar through the hyperstrong scattering medium demonstrates that past failures to discover a pulsar orbiting Sgr A* must inform us about search methodologies and/or the population of GC pulsars.

The National Radio Astronomy Observatory is a facility of the National Science Foun- 
dation operated under cooperative agreement by Associated Universities, Inc. We thank H.J. van Langevelde for useful comments.

\section{REFERENCES}

Armstrong, J. W., Rickett, B. J., \& Spangler, S. R. 1995, ApJ, 443, 209

Backer, D. C. \& Hellings, R. W. 1986, ARA\&A, 24, 537

Bower, G. C., Falcke, H., Herrnstein, R. M., Zhao, J., Goss, W. M., \& Backer, D. C. 2004, Science, 304, 704

Bower, G. C., Goss, W. M., Falcke, H., Backer, D. C., \& Lithwick, Y. 2006, ApJ, 648, L127

Chandran, B. D. G. \& Backer, D. C. 2002, ApJ, 576, 176

Cordes, J. M., Kramer, M., Lazio, T. J. W., Stappers, B. W., Backer, D. C., \& Johnston, S. 2004, New A Rev., 48, 1413

Cordes, J. M. \& Lazio, T. J. W. 1997, ApJ, 475, 557

-. 2002, ArXiv Astrophysics e-prints

Deller, A. T., Brisken, W. F., Phillips, C. J., Morgan, J., Alef, W., Cappallo, R., Middelberg, E., Romney, J., Rottmann, H., Tingay, S. J., \& Wayth, R. 2011, PASP, 123, 275

Deller, A. T., Tingay, S. J., Bailes, M., \& West, C. 2007, PASP, 119, 318

Deneva, J. S., Cordes, J. M., \& Lazio, T. J. W. 2009, ApJ, 702, L177

Desai, K. M. \& Fey, A. L. 2001, ApJS, 133, 395

Eatough, R. P., Falcke, H., Karuppusamy, R., Lee, K. J., Champion, D. J., Keane, E. F., Desvignes, G., Schnitzeler, D. H. F. M., Spitler, L. G., Kramer, M., Klein, B., Bassa, C., Bower, G. C., Brunthaler, A., Cognard, I., Deller, A. T., Demorest, P. B., Freire, P. C. C., Kraus, A., Lyne, A. G., Noutsos, A., Stappers, B., \& Wex, N. 2013a, Nature

Eatough, R. P., Kramer, M., Klein, B., Karuppusamy, R., Champion, D. J., Freire, P. C. C., Wex, N., \& Liu, K. 2013b, in IAU Symposium, Vol. 291, IAU Symposium, 382-384

Fey, A. L., Spangler, S. R., \& Cordes, J. M. 1991, ApJ, 372, 132

Genzel, R., Eisenhauer, F., \& Gillessen, S. 2010, Reviews of Modern Physics, 82, 3121 
Greisen, E. W. 2003, in Astrophysics and Space Science Library, Vol. 285, Astrophysics and Space Science Library, ed. A. Heck, 109

Hotan, A. W., van Straten, W., \& Manchester, R. N. 2004, PASA, 21, 302

Johnston, S., Kramer, M., Lorimer, D. R., Lyne, A. G., McLaughlin, M., Klein, B., \& Manchester, R. N. 2006, MNRAS, 373, L6

Kennea, J. A., Burrows, D. N., Kouveliotou, C., Palmer, D. M., Gögüuss, E., Kaneko, Y., Evans, P. A., Degenaar, N., Reynolds, M. T., Miller, J. M., Wijnands, R., Mori, K., \& Gehrels, N. 2013, ApJ, 770, L24

Kettenis, M., van Langevelde, H. J., Reynolds, C., \& Cotton, B. 2006, in Astronomical Society of the Pacific Conference Series, Vol. 351, Astronomical Data Analysis Software and Systems XV, ed. C. Gabriel, C. Arviset, D. Ponz, \& S. Enrique, 497

Kramer, M., Klein, B., Lorimer, D., Müller, P., Jessner, A., \& Wielebinski, R. 2000, in Astronomical Society of the Pacific Conference Series, Vol. 202, IAU Colloq. 177: Pulsar Astronomy - 2000 and Beyond, ed. M. Kramer, N. Wex, \& R. Wielebinski, 37

Lazio, T. J. W. \& Cordes, J. M. 1998, ApJ, 505, 715

Liu, K., Wex, N., Kramer, M., Cordes, J. M., \& Lazio, T. J. W. 2012, ApJ, 747, 1

Macquart, J.-P., Kanekar, N., Frail, D. A., \& Ransom, S. M. 2010, ApJ, 715, 939

Mori, K., Gotthelf, E. V., Zhang, S., An, H., Baganoff, F. K., Barrière, N. M., Beloborodov, A. M., Boggs, S. E., Christensen, F. E., Craig, W. W., Dufour, F., Grefenstette, B. W., Hailey, C. J., Harrison, F. A., Hong, J., Kaspi, V. M., Kennea, J. A., Madsen, K. K., Markwardt, C. B., Nynka, M., Stern, D., Tomsick, J. A., \& Zhang, W. W. 2013, ApJ, 770, L23

Nord, M. E., Lazio, T. J. W., Kassim, N. E., Goss, W. M., \& Duric, N. 2004, ApJ, 601, L51

Pfahl, E. \& Loeb, A. 2004, ApJ, 615, 253

Rea, N., Esposito, P., Israel, G. L., Papitto, A., Tiengo, A., Baganoff, F., Haggard, D., Mereghetti, S., Burgay, M., Possenti, A., \& Zane, S. 2013, ATeL, \# 5032

Reid, M. J., Menten, K. M., Zheng, X. W., Brunthaler, A., Moscadelli, L., Xu, Y., Zhang, B., Sato, M., Honma, M., Hirota, T., Hachisuka, K., Choi, Y. K., Moellenbrock, G. A., \& Bartkiewicz, A. 2009, ApJ, 700, 137

Roy, S. 2013, ArXiv e-prints 
Shannon, R. M. \& Johnston, S. 2013, ArXiv e-prints

Siemion, A., Bailes, M., Bower, G., Chennamangalam, J., Cordes, J., Demorest, P., Deneva, J., Desvignes, G., Ford, J., Frail, D., Jones, G., Kramer, M., Lazio, J., Lorimer, D., McLaughlin, M., Ransom, S., Roshi, A., Wagner, M., Werthimer, D., \& Wharton, R. 2013, in IAU Symposium, Vol. 291, IAU Symposium, 57-57

Spitler et al., L. 2013, ApJ, submitted

Thompson, C. \& Duncan, R. C. 1995, MNRAS, 275, 255

Trotter, A. S., Moran, J. M., \& Rodriguez, L. F. 1998, ApJ, 493, 666

van Langevelde, H. J., Frail, D. A., Cordes, J. M., \& Diamond, P. J. 1992, ApJ, 396, 686

van Straten, W. \& Bailes, M. 2011, PASA, 28, 1

van Straten, W., Demorest, P., \& Oslowski, S. 2012, Astronomical Research and Technology, 9, 237

Wex, N. \& Kopeikin, S. M. 1999, ApJ, 514, 388

Whitney, A., Kettenis, M., Phillips, C., \& Sekido, M. 2009, in Proceedings of the 8th International e-VLBI Workshop. 22-26 June 2009. Madrid, Spain Published online at http://pos.sissa.it/cgi-bin/reader/conf.cgi?confid=82., p.42, 42-+

Wilkinson, P. N., Narayan, R., \& Spencer, R. E. 1994, MNRAS, 269, 67 

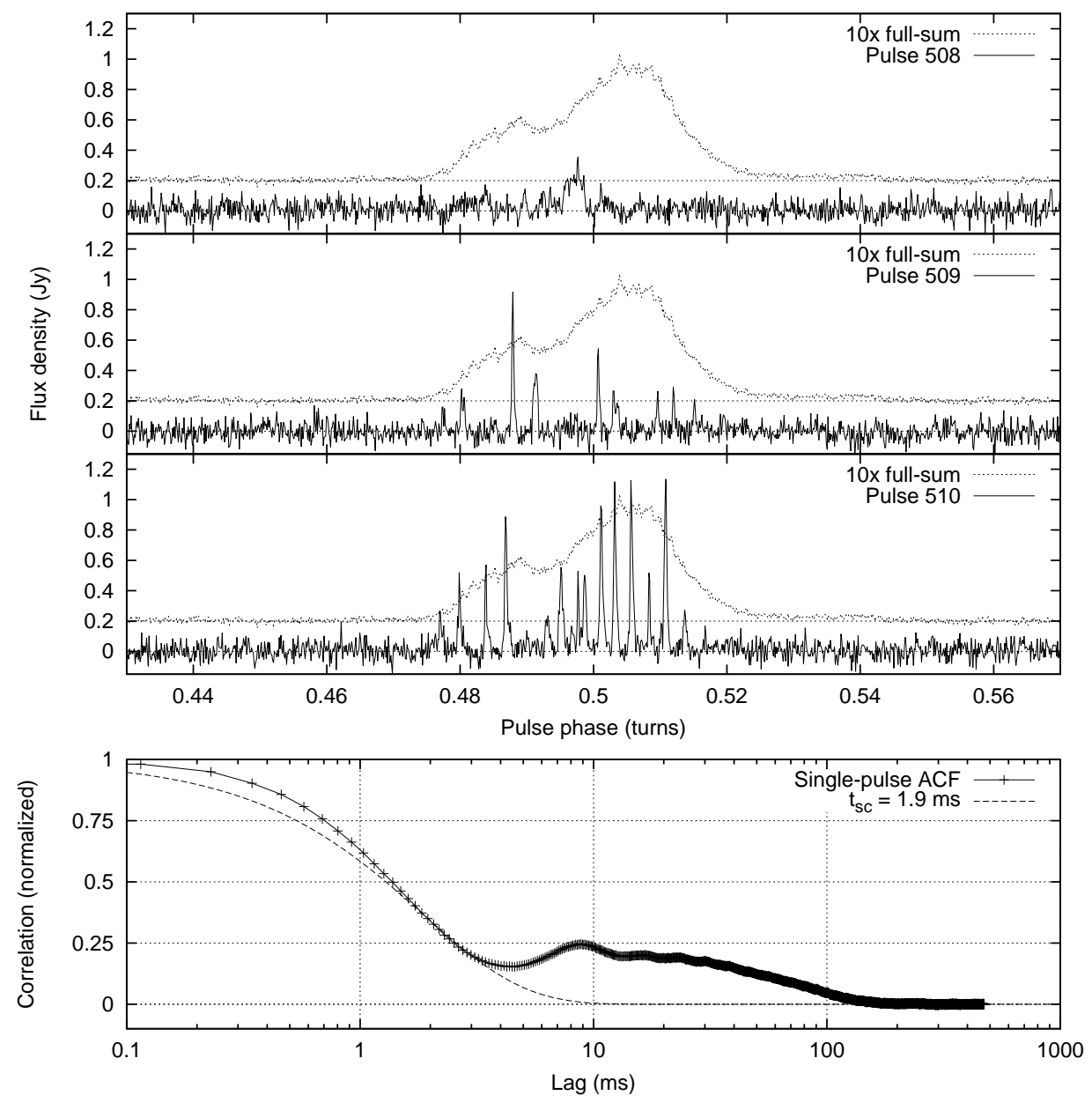

Fig. 1.- Pulse shapes and temporal broadening. The top three panels show a series of three sequential individual pulses from phased VLA observations on MJD 56486. The average pulse profile, scaled by a factor of 10 and offset by $0.2 \mathrm{Jy}$ is also shown for reference. Individual pulses show ms-timescale structure with amplitude $\sim 10$ times the average profile. These often contain quasi-periodically spaced components with a typical separation of $\sim 10 \mathrm{~ms}$ (e.g. pulse number 510 shown here). The bottom panel shows the average single-pulse autocorrelation function, which has a $1 / e$ width of $1.9 \mathrm{~ms}$. The expected ACF of intrinsically narrow single pulses convolved with an exponentially decaying response of $1.9 \mathrm{~ms}$ timescale is shown for comparison. 

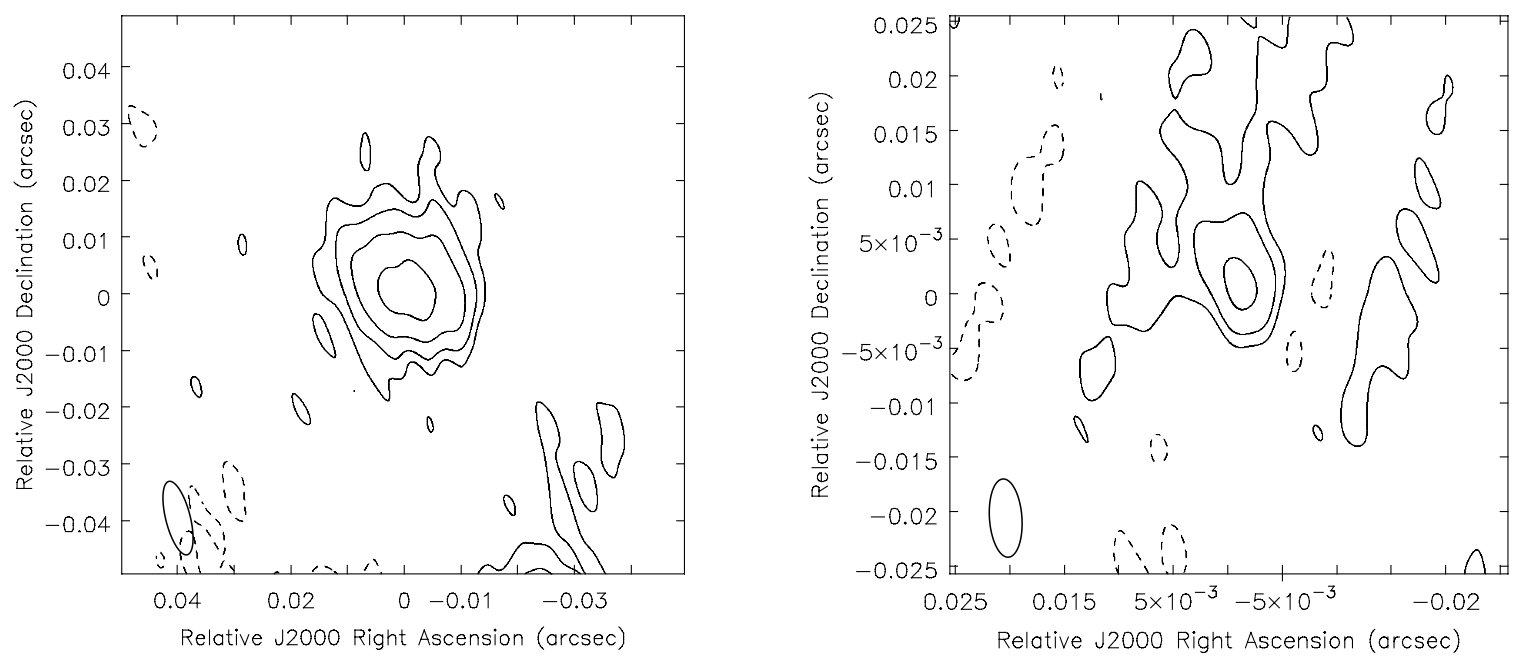

Fig. 2.- Images of SGR J1745-29 at $8.7 \mathrm{GHz}$ (left panel, from the first epoch) and at 15.4 $\mathrm{GHz}$ (right panel, third epoch). Contours are shown at $-2,2,4,8$, and (in the left panel only)16 $\sigma$. The synthesized beam is shown in bold in the lower left corner of the image.

Table 2. Scattered size fits from the VLBI observations

\begin{tabular}{lccc}
\hline \hline $\begin{array}{c}\text { Observation } \\
(\mathrm{MJD})\end{array}$ & $\begin{array}{c}\text { Deconvolved SGR J1745-29 fit } \\
(\mathrm{mas})\end{array}$ & $\begin{array}{c}\text { Deconvolved Sgr A* fit } \\
(\mathrm{mas})\end{array}$ & $\begin{array}{c}\text { VLBI beam } \\
(\mathrm{mas})\end{array}$ \\
\hline \multicolumn{5}{c}{$8.7 \mathrm{GHz}$} \\
\hline 56422 & $16.1_{-1.4}^{+1.3} \times 9.4_{-2.3}^{+2}$ at $87_{-10}^{\circ+12}$ & $16.48_{-0.02}^{+0.02} \times 7.71_{-0.03}^{+0.03}$ at $83.3^{\circ+0.1}$ & $13.3 \times 4.3$ at $14^{\circ}$ \\
56444 & $15.6_{-2.3}^{+2.3} \times 10.1_{-4.5}^{+3.3}$ at $81_{-20}^{\circ+28}$ & $15.9_{-0.1}^{+0.1} \times 6.8_{-0.3}^{+0.2}$ at $89.5_{-0.6}^{\circ+0.6}$ & $17.6 \times 8.1$ at $9^{\circ}$ \\
56486 & $16.1_{-2.8}^{+3.2} \times 10.9_{-5.4}^{+4.1}$ at $71_{-10}^{\circ+23}$ & $16.07_{-0.02}^{+0.01} \times 8.02_{-0.02}^{+0.02}$ at $82.0^{\circ}+0.1$ & $15.0 \times 8.0$ at $6^{\circ}$ \\
Average & $16.0_{-1.1}^{+1.1} \times 9.7_{-1.9}^{+1.6}$ at $82_{-7}^{\circ+10}$ & $16.21_{-0.01}^{+0.01} \times 7.92_{-0.02}^{+0.02}$ at $82.7_{-0.1}^{\circ 0.1}$ & $\ldots$ \\
\hline 5 & $15.4 \mathrm{GHz}$ \\
\hline 56473 & $6.8_{-2.6}^{+2.1} \times 4.4_{-4.4}^{+1.4}$ at $35_{-30}^{\circ+42}$ & $5.44_{-0.01}^{+0.00} \times 3.66_{-0.00}^{+0.01}$ at $81.5_{-0.1}^{\circ+0.1}$ & $7.3 \times 3.0$ at $-5^{\circ}$ \\
\hline
\end{tabular}




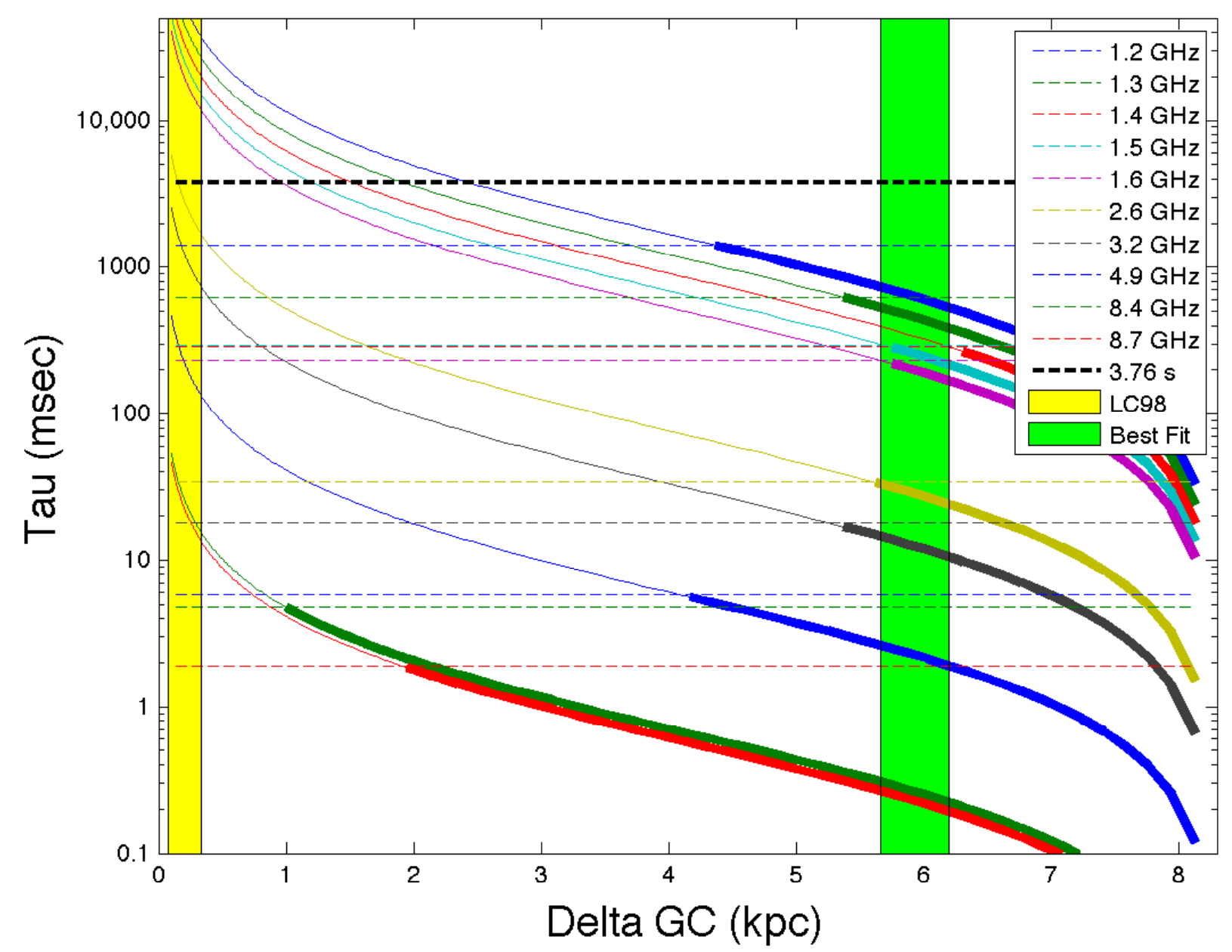

Fig. 3.- Temporal broadening of pulsed radio emission as a function of distance $\Delta$ from the GC. Horizontal dashed lines indicate the measured constraints from Spitler et al. (2013), averaged in $100 \mathrm{MHz}$ bins, and our simultaneous $8.7 \mathrm{GHz}$ VLA observations. Solid lines are theoretical curves based on Equation 1. Permitted parameter space is at values of $\Delta$ where $\tau$ is less than the observed values; these are highlighted as thick solid lines. The dashed black line indicates the pulse period of $3.76 \mathrm{~s}$. The solid yellow region is the solution for $\Delta$ from Lazio \& Cordes (1998). The solid green region is the best-fit solution $\Delta=5.9 \pm 0.3$ kpc from this paper. 